Review Article:

\title{
Etiology and Considerations of Developmental Enamel Defects in Children: A Narrative Review
}

\author{
Prasad Krishnaji Musale $^{1^{*}}$ (D), Abhishek Shrikant Soni² ${ }^{2}$ Sneha Sunil Kothare ${ }^{1}$ (D)
}

1. Department of Pedodontics and Preventive Dentistry, MA Rangoonwala College of Dental Sciences and Research Centre, Pune, Maharashtra, India. 2. Vanilla Smiles Dental Clinic, Pune, Maharashtra, India.

\begin{tabular}{|c|c|}
\hline $\begin{array}{l}\text { Use yourdevice to scan } \\
\text { and read the article online }\end{array}$ & Chtation Musale PK, Soni ASh, Kothare SS. Etiology and Considerations of Developmental Enamel Defects in Children : A Narrative \\
\hline 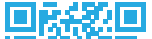 & Review. Journal of Pediatrics Review. 2019; 7(3):141-150. http://dx.doi.org/10.32598/jpr.7.3.141 \\
\hline Hefer & dol ${ }^{8}$ http://dx.doi.org/10.32598/jpr.7.3.141 \\
\hline
\end{tabular}

(c) (1) (5)

Article info:

Received: 14 January 2018

First Revision: 01 May 2018

Accepted: 15 May 2018

Published: 01 July 2019

Key Words:

Developmental defects, Enamel, Primary teeth, Narrative review

\begin{abstract}
A B S T R A C T
Context: Dental enamel is the hardest and highly mineralized structure in human body. However, Developmental Enamel Defects (DEDs) may occur due to an interplay between multiple factors ranging from genetic inadequacy to environmental insults. Primary enamel defects provoke the local or systemic insults that the child might undergo pre-, peri- and post-natally. Several gene mutations and environmental factors, including systemic illnesses have already been identified that can permanently imprint enamel damage. The DED may appear as enamel hypoplasia or hypomineralization. Clinically, DED often presents problems of aesthetics and stained defects, tooth sensitivity, susceptibility to dental caries, erosion and tooth wear.
\end{abstract}

Evidence Acquisition: An electronic search was conducted on PubMed, Cochrane, ScienceDirect and Clinical Key databases with the focus on articles published since 2000. The following keywords were applied: "Developmental Enamel Defect (DED)", "Enamel hypoplasia", and "Primary teeth".

Results: Managing the enamel defects involves early diagnosis and aesthetic rehabilitation of defective enamel, while maintaining its form and function. This should involve close cooperation between the paediatricians and the paediatric dentists, so that preventive regimens can be institutionalised at the earliest.

Conclusions: Despite our understanding of DED, further research is required to establish accurate clinical diagnosis and successful treatment of such enamel defects.

\section{Context}

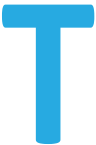

he dental enamel is the hardest and most highly mineralized structure in human body. It consists of over $98 \%$ minerals and less than $2 \%$ of water and organic matrix. It is pro- duced by specialized end-differentiated ameloblasts; the enamel laying cells of the dental organ. Enamel is sequentially laid down over specialized biochemical and cellular pathways. The complex enamel lay-down processes are controlled by genes and influenced by epigenetic and environmental factors. Abnormalities of

* Corresponding Author:

Prasad K Musale, PhD.

Address: Department of Pedodontics and Preventive Dentistry, MA Rangoonwala College of Dental Sciences and Research Centre, Pune, Maharashtra, India. Tel: +91 (98) 22077597

E-mail: pedoprass@gmail.com 
the developmental pathways histologically manifest as neonatal lines and accentuated striae of Retzius (Wilson bands). These result in quantitative defects of the tissue production and or decreased quality of mineralization.

The dental enamel is an essential inert tissue, suited to its role in mastication. The complex enamel specialization process and the life cycle of ameloblasts, which sequentially lays down enamel, explains its high sensitivity to environmental and physiological disorders. The tooth enamel cannot be remodelled after loss due to caries or environmental insults. Thus, any changes in its structure resulting from insults described below will permanently remain on its surface. This may serve as a marker for determining the chronology of the harm (1-3).

Enamel matrix proteins such as amelogenin, ameloblastin and enamelin are secreted in the initial stages of enamel formation, and later stages of mineralization and maturation. DED may be inherited as genetic mutations in the codons that code for the aforementioned proteins, or as a feature of generalized familial conditions. Systemic conditions that involve neuroectodermal derivatives like skin, that share common embryologic origins with teeth, and congenital abnormalities like parathyroid gland disorders that involve mineralization pathways, also show frequent enamel abnormalities. Furthermore, several metabolic conditions, infections, drugs and chemicals as well as trauma and radiation may cause enamel defects due to injury caused by the ameloblasts. Such defects may manifest in the form of:

Hypoplastic enamel defects that result from changes occurring during the matrix formation. Hypomineralization defects resulting from changes that affect the major part of the calcification process. Hypomaturation that refers to changes occurring during mineral accumulation phase (4). Enamel structure defects can develop in the pre-, neo-, and post-natal periods (2). The extension of enamel defect depends on the intensity of etiological factor as well as time period over which it was present during crown stage of tooth development.

Numerous factors are associated with enamel defects in the deciduous and permanent dentition. These factors can be divided into 2 groups including those that cause localized defects limited to 1 or only a few teeth (e.g. trauma, infections, ankylosis \& irradiation), and factors causing generalized defects affecting the majority of or all teeth. These generalized defects can be inherited or caused by environmental factors. The principle environmental factors are infections; neonatal, endocrine, and nutritional disturbances; haemolytic diseases; external intoxication; and cardiac, renal, and gastrointestinal illnesses (2).

Prematurity and low birth weight were also correlated with enamel defects in primary and permanent dentition. Seow et al. reported an inverse relationship between birth weight and the prevalence of enamel hypoplasia. Whilst, Aine et al. found that enamel defects were associated with prematurity. Slayton et al. reported the prevalence of isolated enamel opacities and hypoplasia in a cohort study on well-nourished 4- to 5-year-old children (5-7). They concluded that $6 \%$ of studied children had at least 1 tooth with enamel hypoplasia, and $27 \%$ presented isolated enamel opacities. Enamel opacities were significantly higher in boys than girls.

Mandibular second molars and maxillary second molars were the most affected with enamel hypoplasia and isolated opacities, respectively. Premature children and those with very low birth weight had higher prevalence of enamel defects in the first permanent molars and lateral incisors than the normal birth weight children (8). Teeth with enamel hypoplasia are more susceptible to caries, as they present retentive areas provoking bacterial plaque accumulation. Meanwhile, hypocalcification (opacity) can lead to rapid progression of dental caries (9).

Enamel matrices of mandibular and maxillary anterior teeth are almost completely formed in an 8-month foetus, in which the cusp tips of those teeth just started to calcify. Most of the anterior teeth enamel matrices of an infant are fully formed at birth. Also, calcification is seen in parts of the primary first and second molars. Maxillary primary teeth show slower calcification accomplishment than mandibular primary teeth. Developmental chronology of the human dentition has been widely propagated and used, as suggested by Lunt and Law. As per this chronology, different enamel defect patterns can be anticipated on tooth surfaces. Theoretically, hypoplastic lesions on both maxillary and mandibular central incisors indicate disturbance occurring in the 13week foetus. Similarly, a disturbance occurring at birth will affect all teeth to a certain degree. Moreover, only primary canines, first molars, secondary molars and first permanent molars would be affected by disturbances occurring within 6 months after birth (10).

Massler, Sarnat and Schour demonstrated that both enamel and dentin yield accurate and permanent records of both normal and pathologic accentuations of 
mineral and general metabolism $(11,12)$. These records can be easily read, by virtue of the rhythmic growth of these tissues at the rate of 2.5 to $6.5 \mu \mathrm{m} / \mathrm{d}$. In enamel hypoplasia, the systemic disturbances are recorded by a cessation of ameloblastic activity. Thus, the dentin and particularly the enamel serve as kymographs in their formative and calcifying stages in which indelible marks of the physiologic or pathologic changes in metabolism are recorded. Frequent speculation has been done with regards to defect widths and breadths. For example, the duration of causal stress causes width of the band defect which is a result of the severity of stress $(3,12)$.

\section{Evidence Acquisition}

An electronic search was conducted on PubMed, Cochrane, ScienceDirect and Clinical Key databases. We considered the following keywords: "Developmental Enamel Defect (DED)" , "Enamel hypoplasia", and "Primary teeth". No specific inclusion or exclusion criteria were applied in this review. We included articles published after the year 2000 with respect to DED. However, this was not a limitation during the search.

\section{Results}

This narrative study aims to examine the association between the following issues: 1 . Hypoplasia as a quantitative defect presenting as grooves, pitting, thin or missing enamel; or hypomineralization, which presents as soft enamel due to reduced mineralization or hypomaturation; as altered translucency, either affecting the entire tooth or a localized area known as an opacity in the prismless enamel of the primary teeth; and 2. mother-child conditions; in particular prematurity and low birth weight.

\subsection{DED and maternal variables}

Studies on LBW children have concluded that the reason for enamel hypoplasia in primary teeth and palatal deformities are related to local factors such as overuse of laryngoscope and prolonged use of orotracheal intubation. Other systemic factors such as immaturity, LBW, respiratory distress, rickets of prematurity, neonatal asphyxia, hyperbilirubinemia, neonatal infection and maternal conditions including preeclampsia and diabetes may also cause DED in children. The laying down of the enamel matrix and its subsequent calcifications begin on the 14th gestational week and continue until several months after birth (13-17).
This prolonged pre- and post-natal development period makes the primary enamel susceptible to developmental defects. This process begins at the highest point of the crown and progresses cervically downwards in incremental layers towards the tooth neck. The enamel lacks ability to defend and repair itself. Any systemic circumstances that disrupts its structural integrity causes permanent structural defect in the developing teeth. The modified Developmental Defects of Enamel (DDE) Index presented by the Federation Dentaire Internationale (FDI) Commission on Oral Health was used to register DED (18).

Younger maternal age, as evidenced by the mother's medical and reproductive history was a predictor of DDE in children. Other variables included maternal schooling, mother's BMI, mother suffering from systemic illnesses like gestational diabetes, and infants who were not breastfed showed higher incidence of developmental defects. The composition of maternal milk, with its nutrients available in appropriate quantity and quality for the normal growth and development of the child, i.e. the formation of the dental organ, could initially explain these findings.

Another possible explanation is based on the immunological and anti-infectious properties of human milk in the reduction of illnesses, especially during the first year after birth. According to Nóren et al., the mineralization of primary teeth is being completed in the first year of life (19). There was a statistically significant association between the incidence of enamel defects and tobacco use during pregnancy, with a positive linear relationship between the number of cigarettes smoked per day and the prevalence of hypoplastic defects. Similarly, children born by normal vaginal delivery were unlikely to develop hypoplasia, compared to those born by caesarean delivery. Lack of prenatal care during the first trimester and elevated blood lead levels may also contribute to DEDs (16). The following points suggest that an anticipatory guidance be established between health care providers and expectant mothers.

\subsection{Prematurity and low birthweight in infants}

Regardless of severity of prematurity or low birthweight, preterm infants have increased risk of shortterm and long-term complications, including cerebral palsy, neurodevelopmental complications, and chronic medical needs compared with their full-term counterparts. Effects of preterm birth on oral structures vary among infants, depending on several factors, such as gestational age, birthweight, postpartum medical com- 
plications and interventions, as well as growth and developmental complications. The risks of certain orodental manifestations are higher among preterm infants compared with full-term ones (20).

The prevalence of developmental enamel defects may be up to $96 \%$ among preterm and or very low birthweight and extremely low birthweight infants. Enamel defects are associated with local trauma and calcium homeostatic imbalance during the prenatal and postnatal periods, leading to disturbances during enamel matrix formation and mineralization. Chemical analysis of primary teeth indicated that the calcium/carbon ratio of the enamel surfaces was significantly lower (therefore more porous) in preterm infants compared with fullterm controls (21).

Scanning electron microscopy analyses have concurred that the enamel of preterm infants is thinner and malformed compared to those completing gestational age of 38-40 weeks. Even postnatally formed enamel could not adequately compensate pre-natal enamel (22). Merheb et al. reported that the occurrence of enamel hypoplasia is significantly higher among very low-birthweight infants with lower serum phosphorous levels (23). Etiologic factors attributing to the development of enamel defects among preterm infants include disruption to amelogenesis and enamel matrix formation and mineralization in utero and postnatal development, especially in the presence of stress, intrauterine or extrauterine growth restriction, maternal systemic illnesses, use of medications during pregnancy, postnatal infant systemic illnesses and medications, metabolic derangements during and after the neonatal period, and local trauma $(20,24,25)$.

Enamel defects among preterm infants were specifically attributed to localized trauma associated with laryngoscope, endotracheal intubation, and oral or nasogastric tube, in the past. The primary maxillary left incisors were the most commonly affected ones. Oral intubation; however, reduces the occurrence of enamel defects due to this issue $(8,20)$. Risks of enamel defects affecting both primary and permanent teeth are further increased by fever, malnutrition, dental trauma, infections, medical conditions and neonatal complications involving use of medications $(26,27)$.

The potential increased risk for caries among preterm infants may be due to factors that modify the oral flora and demineralization versus re-mineralization equilibrium such as medical conditions and medications, immature or impaired immunity, foetal growth retardation, enamel defects, feeding and dietary factors, and cognitive and behavioural factors, e.g. immature immunity may facilitate early colonization of cariogenic bacteria, like Streptococcus mutans $(8,28)$.

Enamel defects may facilitate early colonization of cariogenic bacteria due to roughened tooth surfaces. They could also facilitate structural breakdown due to reduced enamel quantity and quality (29). Preterm infants are more likely to require medications for a prolonged period of time which may be acidic and or contain a high percentage of sucrose, thereby promoting the establishment of a more acidogenic and cariogenic oral flora $(29,30)$. Transmission-related behaviours, such as increased maternal contact during feeding, may also increase possibility of earlier colonization of cariogenic bacteria among predentate preterm infants (31). In addition, weight gain is often more important for preterm infants than their full-term counterparts, resulting in increased odds of on-demand feeding, frequent feeding, night feeding, and the consumption of high-calorie infant formula that are often higher in sugar content. Primary tooth crown dimensions reduce by approximately $10 \%$ in preterm infants. Developmental enamel defects increase the odds of hypersensitivity due to dentine exposure, tooth wear, and if anteriorly located, aesthetic concerns due to dental caries (8).

Developmental enamel defects in the primary dentition exponentially increase the odds of early childhood caries, more specifically "hypoplasia-associated early childhood caries (HAS-ECC)". In addition, given that enamel formation of the first permanent molars and incisors occurs simultaneous to that of second primary molars, the presence of enamel defects on the primary second molars indicates the need for more frequent follow-up because of the increased risk of molar-incisor hypoplasia in the permanent dentition $(8,32)$. However, establishing a dental home at the earliest with a paediatric dentist can benefit the overall oral health in preterm and very low birthweight infants.

\subsection{DED and neonatal variables}

Children with low 5-min Apgar scores and those who received parenteral nutrition during the neonatal period showed hypoplastic defects. Combined defects were frequently observed in children with low 1- and 5-min Apgar scores and neonatal acidosis. A higher prevalence of severe defects (i.e. combined defects) has been noted in children requiring orotracheal intubation and mechanical ventilation in the neonatal period. Maxillary teeth are more commonly and severely affected than 
their counterparts in the mandibular arch. Moreover, children with LBW who were not intubated show defects that are symmetrically distributed, whereas they are located asymmetrically more often on the left in those who required intubation (8).

Respiratory distress syndrome was diagnosed according to the established criteria and defined as follows: 1. Mild (infant requiring only supplemental oxygen); 2. Moderate (necessarily requiring nasal constant positive pressure); and 3. Severe (when respiratory support included mechanical ventilation and endotracheal intubation).

DED was significantly more frequent among those with health problems during the first year of life (33).

\subsection{Inherited conditions involving enamel formation}

\subsubsection{Amelogenesis Imperfecta}

Amelogenesis Imperfecta (AI) addresses hereditary defects of enamel, not associated with defects in other parts of the body or other health problems. The enamel defects are highly variable and include abnormalities, classified as hypoplastic, hypomaturation, and hypocalcified, depending on the stage of enamel formation that is affected by the genetic defect. The prevalence rates of it varies approximately between 1:1000 and 1:16000 in different populations (26).

The formation of this highly organised and unusual structure is rigorously controlled in ameloblasts through the interaction of a number of organic molecules that include enamelin, amelogenin, ameloblastin, tuftelin, amelotin, and Dentine Sialophosphoprotein (DSPP) enzymes such as kallikrein and Matrix Metalloproteinase 20 (MMP20). Any mutations in these proteins can cause Al. Mutation of the gene encoding the enamel-specific aforementioned proteins are associated with some of the following defects and their responsible proteins: Hypoplasia (Surface pits, thin enamel): AMEL and ENAM ${ }^{27}$; X-linked AI: AMELX, MMP20, WDR72; Hypomineralization, Hypomaturation: KLK4, MMP20, WDR72; Autosomal dominant hypomineralization: FAM83H.

The phenotypic variation among individuals within a family having the same mutation is well known in $\mathrm{Al}$, which results from differences in gene expression. Furthermore, vertical grooving on the surface of the tooth with bands of normal enamel alternating with deficient enamel areas is observed in females affected with $\mathrm{X}$ - linked Al. While, males in the same family show complete enamel absence $(34,35)$.

\subsection{Defects Of epithelial tissues causing enamel defects}

A lot of inherited syndromes, in particular those that involve ectodermal derivatives skin, hair and nail are usually presented with DED. Since all these structures have a frequent embryonic mutation in common genes result in abnormalities seen in all tissues. Congenital erythropoietic porphyria, ectodermal dysplasia, tuberous sclerosis and Epidermolysis Bullosa (EB) are among the dermatological conditions in which DED have been reported. The frequency ranges from $8.6 \%$ in recessive dystrophic EB to $100 \%$ in junctional EB. Haemolytic anaemia, hypertrichosis, skin fragility, photosensitivity, and red-brown porphyrin pigmentation of bones and discoloured and hypoplastic teeth are detected in congenital erythropoietic porphyria $(27,36)$.

Striking enamel defects are usually observed in TrichoDento-Osseous (TDO) syndrome. It is an autosomal dominant condition caused by mutations in the DLX3 homeobox gene. The characteristics of TDO comprise severe hypomineralization of the enamel, taurodontism, and abnormalities in nails, hair and bones (37).

\subsection{Mineralization pathway defects causing enamel defects}

The mineralization pathways at some stages involving the parathyroid glands also show abnormalities of enamel development. Many inherited conditions such as the velocardiofacial syndrome or DiGeorge syndrome or 22q11.2 deletion syndrome show enamel hypomineralization and hypoplasia. Rare congenital conditions such as the Kenny-Caffey syndrome and the autoimmune polyendocrinopathy-candidiasis-ectodermal dystrophy syndrome have strongly associated with hypoparathyroidism and enamel defects.

The association of DED are also reported with vitamin $D$ deficiency due to genetic metabolic conditions or malnutrition. This condition often results in failure of bone matrix to mineralize as in rickets. Children residing in sunlight-deficient areas are unable to activate provitamin D. Those who do not consume sufficient Vitamin $D$ often suffer from nutritional rickets. Two genetic variants of vitamin D dependent rickets are recognized that cause severe enamel hypoplasia due to hypocalcemia (38). The deficiency of the enzyme 25-hydroxyvitamin D-1alpha hydroxylase which leads to lack of calcitriol synthesis and causes type 1 or pseudovitamin D defi- 
ciency rickets. In contrast, non-responsiveness of the vitamin D receptor causes type 2 Vitamin D dependent rickets (38). Nutritional status is usually calculated according to height and age using the Waterlow index. This index uses WHO international reference population children classified as malnourished, if below under $95 \%$ of the height for age median. Another interesting finding was that the low water intake and early weaning should be included in any longitudinal investigation to be linked as possible aetiological factors $(27,38,39)$.

\subsection{Acquired conditions associated with DED}

In addition to genetic conditions, many environmental and acquired systemic changes can also disturb the formation of enamel. If an insult occurs during enamel matrix secretion, hypoplastic defects are likely to occur, in contrast to an insult occurring during the mineralization stages which usually produces hypomineralization defects (27).

\subsubsection{Systemic conditions}

Numerous metabolic disturbances, infections, chemicals and drugs are associated with DED. Local causes include trauma, radiation and localised infection. Since enamel lacks its inherent capacity to repair itself the location of the enamel defect indicates the approximate time, duration and intensity of the insult in relation to the chronology of tooth development. The evidence in support of the following statement has been derived from clinical cases and epidemiological studies. Some of the reports have claimed an association between factors causing organ damage and DED. A strong correlation between DED and children suffering from cerebral palsy has been observed. Whilst, systemic disturbances such as foetal anoxia, infections and hyperbilirubinemia damage both the enamel and the development of the brain cells.

Prenatal factors including vitamin D deficiency, maternal smoking and neonatal tetany, and nutritional deficiencies during the postnatal period may contribute to DED (40-42). A premature child suffers from respiratory immaturity, cardiovascular, gastrointestinal and renal abnormalities, intracranial haemorrhage and anaemia. The additive effect of these problems result in DED in premature children. Furthermore, defects found in preterm children usually emerge from adverse systemic conditions associated with premature birth, such as hypocalcaemia, osteopenia and hyperbilirubinemia $(5,43,44)$.
The inability of the immature gastrointestinal tract to absorb calcium and phosphorus minerals also contributes to enamel hypoplasia in preterm children (44). Local trauma from laryngoscopy and endotracheal intubation to manage respiratory distress, increase the risks for damage to the developing enamel in primary maxillary incisor teeth $(19,45)$. Coeliac disease associated with gut enteropathy in children causes malabsorption and mineral deficiencies due to gluten intolerance. This leads to low serum calcium concentration during enamel formation. When gluten is introduced in children suffering from IgE mediated gluten sensitivity, the IgE could enter the maturing enamel and inhibit its full maturation, thus increase diffuse opacities $(27,46)$. Chronic renal and liver diseases result in disruption of mineralization pathways that places affected children at risk for enamel defects (47-49). Prolonged bouts of fever and infections caused by microorganisms during the neonatal period may infect children's developing ameloblasts via metabolic products that may directly or indirectly alter cellular processes.

Clinical reports have suggested that viral infections such as chickenpox, rubella, measles, mumps, influenza and cytomegalovirus, infections of the urinary tract, otitis, upper respiratory diseases and congenital syphilis are well known causes of enamel hypoplasia in primary and permanent dentition $(15,50)$. Excessive fluoride use, tetracycline use before the age of 8 years, and other cytotoxic drugs have been implicated to cause enamel damage. These result from the direct effects of fluoride on the developing ameloblasts. Characteristics and prevalence of primary tooth fluorosis in these subjects have been reported separately.

Fluorosis and non-fluoride (isolated) opacities were differentiated using Russell's Periodontal Index and the Developmental Defects of Enamel (DDE) index (27, 51, 52). The enamel lesions are differentiated based on the shape, colour, extent of the lesion, and the affected teeth. If the smooth surface lesions of the tooth are creamy-yellow to brown in colour, and well demarcated, they are implicated as non-fluoride opacities. In contrast, fluorosis lacks well-defined margins, and is more diffuse and symmetrical, with white patches or lines. Environmental exposure to lead paint, or accidental orpica ingestion may cause bilateral enamel hypoplasia $(6,53)$. Amoxicillin was developed in the 1960s. It is a bacteriolytic $\beta$-lactam antibiotic in the aminopenicillin family, used to treat Gram-positive and Gram-negative bacteria. It diffuses easily into tissues and body fluids. It may cross the placenta and is excreted into breast milk in small quantities. 
Amoxicillin use in the early years of postnatal life seems to be linked to fluorosis-like enamel defects on maxillary central incisors. Considering the developmental stages of enamel formation of maxillary central incisors, amoxicillin affects the ameloblasts during their secretory phase. It has also been attributed that amoxicillin use could reduce gene expression of amelogenins and other matrix proteins. In addition, it can decrease the activity of proteinases that hydrolyse matrix proteins. Although there is some evidence that amoxicillin can cause enamel defects, it is difficult to isolate the effects of the fevers and infections which had necessitated the use of these antibiotics. However, further studies are required to explore the exact involved mechanism $(54,55)$.

\section{Conclusion}

Oral health care for children with DED should start as early as possible to enable early risk assessment, detection, and management of oro-dental anomalies and prevention of acquired oral conditions, through the establishment of a dental home. Parents and caregivers of pre-term children must be provided with timely advice and support regarding oral health in the context of general health, growth, and well-being. These goals are best achieved interprofessionally with the help of nondental health practitioners.

\section{Ethical Considerations}

\section{Compliance with ethical guidelines}

There is no ethical principle to be considered doing this research.

\section{Funding}

This research did not receive any specific grant from funding agencies in the public, commercial, or not-forprofit sectors.

\section{Authors contributions}

Literature search: Prasad Krishnaji Musale and Sneha Sunil Kothare; Compiled the manuscript: Prasad Krishnaji Musale and Abhishek Shrikant Soni; and read and approved the final manuscript: All three authors.

\section{Conflicts of interest}

The authors declare no conflict of interest.

\section{References}

1. Seow WK. Clinical diagnosis of enamel defects: Pitfalls and practical guidelines. International Dental Journal. 1997; 47(3):173-82. [DOI:10.1002/j.1875-595X.1997.tb00783.x ]

2. Small BW, Murray JJ. Enamel opacities: Prevalence, classifications, and aetiological considerations. Journal of Dentistry. 1978; 6(1):33-42. [DOI:10.1016/0300-5712(78)90004-0]

3. Goodman HA, Rose JC. Assessment of systemic physiological perturbations from dental enamel hypoplasias and associated histological structures. Yearbook of Physical Anthropology. 1990; 33(s11):59-110. [DOI:10.1002/ ajpa.1330330506]

4. Lunardelli SE, Peres MA. Breast-feeding and other motherchild factors associated with developmental enamel defects in the primary teeth of Brazilian children. Journal of Dentistry for Children. 2006; 73(2):70-8.

5. Seow WK, Humphrys C, Tudehope DI. Increased prevalence of developmental dental defects in low birth-weight, prematurely born children: A controlled study. Journal of Pediatric Dentistry. 1987; 9(3):221-5.

6. Aine L, Backström MC, Mäki R, Kuusela AL, Koivisto AM, Ikonen RS, et al. Enamel defects in primary and permanent teeth of children born prematurely. Journal of Oral Pathology \& Medicine. 2000; 29(8):403-9. [DOI:10.1034/j.16000714.2000.290806.x]

7. Slayton RL, Warren JJ, Kanellis MJ, Levy SM, Islam M. Prevalence of enamel hypoplasia and isolated opacities in the primary dentition. Pediatric Dentistry. 2001; 23(1):32-43.

8. Tsang AK. The special needs of preterm children-an oral health perspective. Dental Clinics. 2016; 60(3):737-56. [DOI:10.1016/j.cden.2016.02.005]

9. Seow WK. Oral complications of premature birth. Australian Dental Journal. 1986; 31(1):23-9. [DOI:10.1111/j.1834-7819.1986. tb02979.x]

10. Lunt RC, Law DB. A review of the chronology of eruption of deciduous teeth. The Journal of the American Dental Association. 1974; 89(4):872-9. [DOI:10.14219/jada.archive.1974.0484]

11. Massler M, Schour I, Poncher HG. Developmental pattern of the child as reflected in the calcification pattern of the teeth. American Journal of Diseases of Children. 1941; 62(1):3367. [DOI:10.1001/archpedi.1941.02000130042004]

12. Sarnat BG, Schour I. Enamel hypoplasia (chronologic enamel aplasia) in relation to systemic disease: A chronologic, morphologic and etiologic classification. The Journal of the American Dental Association. 1941; 28(12):1989-2000. [Doi:10.14219/jada.archive.1941.0307]

13. Agarwal KN, Narula S, Faridi MM, Kalra N. Deciduous dentition and enamel defects. Indian Pediatrics. 2003; 40(2):124-9. 
14. Franco KM, Line SR, Moura Ribeiro MV. Prenatal and neonatal variables associated with enamel hypoplasia in deciduous teeth in low birth weight preterm infants. Journal of Applied Oral Science. 2007; 15(6):518-23. [DOI:10.1590/ S1678-77572007000600012]

15. Seow WK. Enamel hypoplasia in the primary dentition: A review. ASDC journal of Dentistry for Children. 1991; 58(6):441-52.

16. Vello MA, Martínez Costa C, Catala M, Fons J, Brines J, Guijarro Martínez R. Prenatal and neonatal risk factors for the development of enamel defects in low birth weight children. Oral Diseases. 2010; 16(3):257-62. [DOI:10.1111/ j.1601-0825.2009.01629.x]

17. Moore KL, Persaud TVN, Torchia M. The Developing Human. Philadelphia: Saunders; 2015

18. Commission on Oral Health, Research and Epidemiology. An epidemiological index of developmental Defects of Dental Enamel (DDE Index). International Dental Journal. 1982; 32(2):159-67.

19. Nóren JG, Ranggard L, Klingberg G, Persson C, Nilsson K. Intubation and mineralization disturbances in the enamel of primary teeth. Acta Odontologica Scandinavica. 1993; 51(5):271-5. [PMID]

20. Nelson S, Albert JM, Geng C, Curtan S, Lang K, Miadich S, et al. Increased enamel hypoplasia and very low birthweight infants. Journal of Dental Research. 2013; 92(9):788-94. [DOI: 10.1177/0022034513497751] [PMID] [PMCID]

21. Rythén M, Sabel N, Dietz W, Robertson A, Norén JG. Chemical aspects on dental hard tissues in primary teeth from preterm infants. European Journal of Oral Sciences. 2010; 118(4):38995. [DOI:10.1111/j.1600-0722.2010.00755.x] [PMID]

22. Seow WK, Young WG, Tsang AK, Daley T. A study of primary dental enamel from preterm and full-term children using light and scanning electron microscopy. Pediatric Dentistry. 2005; 27(5):374-9. [PMID]

23. Merheb R, Arumugam C, Lee W, Collin M, Nguyen C, Groh Wargo $S$, et al. Neonatal serum phosphorus levels and enamel defects in very low birth weight infants. Journal of Parenteral and Enteral Nutrition. 2016; 40(6):835-41. [doi:10.1034/j.1600-0714.2000.290806.x]

24. Pinho JR, Thomaz EB, Lamy ZC, Libério SA, Ferreira EB. Are low birth weight, intrauterine growth restriction, and preterm birth associated with enamel developmental defects? Pediatric Dentistry. 2012; 34(3):244-8. [PMID]

25. Correa Faria P, Martins Junior PA, Viieira Andrade RG, Oliveira Ferreira FE, Marques LS, Ramos-Jorge MI. Developmental defects of enamel in primary teeth: Prevalence and associated factors. International Journal of Paediatric Dentistry. 2013; 23:173-9. [DOI:10.1111/j.1365263X.2012.01241.x]

26. Salanitri S, Seow WK. Developmental enamel defects in the primary dentition: Aetiology and clinical manage- ment. Australian Dental Journal. 2013; 58(2):133-40. [Doi:10.1111/adj.12039]

27. Seow WK. Developmental defects of enamel and dentine: challenges for basic science research and clinical management. Australian Dental Journal. 2014; 59(Suppl. 1):14354. [DOI:10.1111/adj.12104]

28. Wan AK, Seow WK, Purdie DM, Bird PS, Walsh L, Tudehope DI. Oral colonization of Streptococcus mutans in six-monthold predentate infants. Journal of Dental Research. 2001; 80(12):2060-5. [DOI:10.1177/00220345010800120701]

29. Oliveira AF, Chaves AM, Rosenblatt A. The influence of enamel defects on the development of early childhood caries in a population with low socioeconomic status: A longitudinal study. Caries Research. 2006; 40(4):296-302. [DOI:10.1159/000093188]

30. Bigeard L. The role of medication and sugars in pediatric dental patients. Dental Clinics of North America. 2000; 44(3):443-56.

31. Fontana M, Jackson R, Eckert G, Swigonski N, Chin J, Zandona $A F$, et al. Identification of caries risk factors in toddlers. Journal of Dental Research. 2011; 90(2):209-14. [DOI:10.1177/0022034510385458]

32. Caufield PW, Li Y, Bromage TG. Hypoplasia-associated severe early childhood caries- A proposed definition. Journal of Dental Research. 2012; 91(6):544-50 [DOI:10.1177/0022034512444929]

33. Klaus MH, Fanaroff AA. Care of the high risk neonate. Philadelphia: Saunders; 1979.

34. Coxon TL, Brook AH, Barron MJ, Smith RN. Phenotypegenotype correlations in mouse models of amelogenesis imperfect caused by AMELX and ENAM mutations. Cells Tissues Organs. 2012; 196(5):420-30. [DOI:10.1159/000336440]

35. Wright JT, Hart TC, Hart PS, Simmons D, Suggs C, Daley B, et al. Human and mouse enamel phenotypes resulting from mutation or altered expression of AMEL, ENAM, MMP20 and KLK4. Cells Tissues Organs. 2009; 189(1-4):224-9. [DOI:10.1159/000151378]

36. Freiman A, Borsuk D, Barankin B, Sperber GH, Krafchik B. Dental manifestations of dermatologic conditions. Journal of the American Academy of Dermatology. 2009; 60(2):289-98. [DOI:10.1016/j.jaad.2008.09.056]

37. Nieminen $P$, Lukinmaa $P L$, Alapulli $H$, Methuen $M$, Suojärvi T, Kivirikko $S$, et al. DLX3 homeodomain mutations cause tricho-dento-osseous syndrome with novel phenotypes. Cells Tissues Organs. 2011; 194(1):49-59. [DOI:10.1159/000322561]

38. Malloy PJ, Feldman D. Genetic disorders and defects in vitamin D action. Rheumatic Disease Clinics. 2012; 38(1):93-106

39. Patzer L, van't Hoff W, Dillon MJ. X-linked hypophosphataemic rickets: Recognition, treatment and prognosis. Cur- 
rent Paediatrics. 1998; 8(1):26-30. [DOI:10.1016/S09575839(98)80055-9]

40. Seow WK, Ford D, Kazoullis S, Newman B, Holcombe T. Comparison of enamel defects in the primary and permanent dentitions of children from a low-fluoride District in Australia. Pediatric Dentistry. 2011; 33(3):207-12.

41. Herman SC, McDonald RE. Enamel hypoplasia in cerebral palsied children. Journal of Dentistry for Children. 1963; 30:46-9.

42. Martinez A, Cubillos $P$, Jim enez $M$, Brethauer U, Catal an $P$ Gonz alez U. Prevalence of developmental enamel defects in mentally retarded children. Journal of Dentistry for Children. 2002; 69:151-5.

43. Seow WK, Brown JP, Tudehope IA, O'Callaghan M. Dental defects in the deciduous dentition of premature infants with low birthweight and neonatal rickets. Pediatric Dental Journal. 1982; 6(20):89-92.

44. Seow WK, Masel JP, Weir C, Tudehope DI. Mineral deficiency in the pathogenesis of enamel hypoplasia in prematurely born, very low birthweight children. Pediatric Dental Journal. 1989; 11(4):297-302. [PMID]

45. Seow WK, Brown JP, Tudehope DI, O'callaghan M. Developmental defects in the primary dentition of low birth-weight infants: Adverse effects of laryngoscopy and prolonged endotracheal intubation. Pediatric Dental Journal. 1984; 6(1):28-31.

46. Majorana A, Bardellini E, Ravelli A, Plebani A, Polimeni A, Campus G. Implications of gluten exposure period, $C D$ clinical forms, and HLA typing in the association between celiac disease and dental enamel defects in children. A case-control study. International Journal of Paediatric Dentistry. 2010; 20(2):119-24. [DOI:10.1111/ j.1365-263X.2009.01028.x]

47. Koch MJ, Bührer R, Pioch T, Schärer K. Enamel hypoplasia of primary teeth in chronic renal failure. Pediatric Nephrology. 1999; 13(1):68-72. [DOI:10.1007/s004670050566]

48. Oliver WJ, Owings CL, Brown WE, Shapiro BA. Hypoplastic enamel associated with the nephrotic syndrome. Pediatrics. 1963; 32(3):399-406.

49. Seow WK, Shepherd RW, Ong TH. Oral changes associated with end-stage liver disease and liver transplantation: Implications for dental management. Journal of Dentistry for Children. 1991; 58(6):474-80.

50. Kliegman R, Nelson WE. Nelson textbook of pediatrics. Philadelphia: Saunders; 2011. [DOI:10.1016/B978-1-43770755-7.00210-4]

51. Bronckers AL, Lyaruu DM, DenBesten PK. The impact of fluoride on ameloblasts and the mechanisms of enamel fluorosis. Journal of Dental Research. 2009; 88(10):877-93. [DOI: 10.1177/0022034509343280]
52. Seow WK. Trichodentoosseous (TDO) syndrome: Case report and literature review. Pediatric Dental Journal. 1993; 15(5):355-61.

53. Owen LN. The effects of administering tetracyclines to young dogs with particular reference to localization of the drugs in the teeth. Archives of Oral Biology. 1963; 8(6):71527. [DOI:10.1016/0003-9969(63)90003-7]

54. Hong L, Levy SM, Warren JJ, Dawson DV, Bergus GR, Wefel JS. Association of amoxicillin use during early childhood with developmental tooth enamel defects. Archives of Pediatrics \& Adolescent Medicine. 2005; 159(10):943-8. [DOI:10.1001/archpedi.159.10.943] [PMID]

55. Hong L. Association of amoxicillin use during early childhood with developmental tooth enamel defects. Journal of Public Health Dentistry. 2011; 71(3):229-35. 
This Page Intentionally Left Blank 